\title{
Can $f 1$ levels in hair and homes of different dog breeds: Lack of evidence to describe any dog breed as hypoallergenic
}

\author{
Doris W. Vredegoor, BSc, ${ }^{a}$ Ton Willemse, $\mathrm{PhD},{ }^{\mathrm{b}}$ Martin D. Chapman, PhD, ${ }^{\mathrm{c}}$ Dick J. J. Heederik, PhD, ${ }^{\mathrm{a}}$ and \\ Esmeralda J. M. Krop, PhD $^{\mathbf{a}}$ Utrecht, The Netherlands, and Charlottesville, Va
}

Background: Certain dog breeds are described and marketed as being "hypoallergenic" on the basis of anecdotal reports that these dogs are better tolerated by patients allergic to dogs. Objective: These observations were investigated by comparing Can $\mathbf{f} 1$ (major dog [Canis familiaris] allergen) levels in hair and coat samples and in the home environment of various hypoallergenic (Labradoodle, Poodle, Spanish Waterdog, and Airedale terrier) and non-hypoallergenic dogs (Labrador retriever and a control group).

Methods: Hair and coat samples were obtained from dogs, and settled floor and airborne dust samples were taken from the dogs' homes. Can $f 1$ concentrations were measured by using ELISA, and results were analyzed by using multiple linear regression analyses.

Results: Significantly higher Can $\mathbf{f} 1$ concentrations were found in hair and coat samples of hypoallergenic dogs $(n=196$, geometric mean [GM], $2.26 \mu \mathrm{g} / \mathrm{g}$, geometric standard deviation [GSD], 0.73, and GM, 27.04 $\mu \mathrm{g} / \mathrm{g}$, GSD, 0.57, respectively) than of non-hypoallergenic dogs $(\mathrm{n}=160, \mathrm{GM}, 0.77 \mu \mathrm{g} / \mathrm{g}, \mathrm{GSD}, 0.71$, and GM, $12.98 \mu \mathrm{g} / \mathrm{g}$, GSD, 0.76, respectively). Differences between breeds were small, relative to the variability within a breed. Can $\mathrm{f} 1$ levels in settled floor dust samples were lower for Labradoodles, but no differences were found between the other groups. No differences in airborne levels were found between breeds.

Conclusion: So-called hypoallergenic dogs had higher Can $f$ 1 levels in hair and coat samples than did control breeds. These differences did not lead to higher levels of environmental exposure to dog allergens. There is no evidence for the classification of certain dog breeds as being "hypoallergenic." (J Allergy Clin Immunol 2012;130:904-9.)

From ${ }^{a}$ the Division of Environmental Epidemiology, Institute for Risk Assessment Sciences, and ${ }^{b}$ the Department of Clinical Sciences of Companion Animals, Faculty of Veterinary Medicine, Utrecht University, Utrecht; and ${ }^{c}$ Indoor Biotechnologies, Inc, Charlottesville.

This work was supported by a grant from the Honours Program of the Faculty of Veterinary Medicine of Utrecht University, a research program for students. Indoor Biotechnologies, Inc, Charlottesville, Va, provided the Can $\mathrm{f} 1$ kit used in this study.

Disclosure of potential conflict of interest: M. D. Chapman has an ownership position with Indoor Biotechnologies, Inc, and Indoor Biotechnologies, Ltd; has received research support from the National Institute for Environmental Health Sciences and the National Institute for Allergy and Infectious Diseases; and is on the Virginia Biotechnology Association board of directors. E. J. M. Krop acknowledges that Indoor Biotechnologies provided the kit for this study. The rest of the authors declare that they have no relevant conflicts of interest.

Received for publication December 14, 2011; revised May 7, 2012; accepted for publication May 16, 2012

Available online June 22, 2012.

Corresponding author: Esmeralda J. M. Krop, PhD, Division of Environmental Epidemiology, Institute for Risk Assessment Sciences, Utrecht University, PO Box 80178, 3508 TD, Utrecht, The Netherlands. E-mail: e.j.m.krop@uu.nl.

$0091-6749 / \$ 36.00$

(C) 2012 American Academy of Allergy, Asthma \& Immunology

doi:10.1016/j.jaci.2012.05.013
Key words: Can $f$ 1, dog allergen, dog breeds, environmental exposure, hair, hypoallergenic dogs

Discuss this article on the JACI Journal Club blog: www.jacionline.blogspot.com.

Up to $20 \%$ of the general population in Western countries is allergic to dogs, ${ }^{1}$ and exposure to dog allergens is a significant cause of respiratory and asthma symptoms. ${ }^{2,3}$ Many patients allergic to dogs report that some dog breeds are better tolerated than others. Although scientific evidence is weak, patients believe that these breeds are better tolerated because they shed less or have a compact coat. These dog breeds have anecdotally been described by patients and by dog breeders as being "hypoallergenic." A handful of studies have investigated qualitative or quantitative differences in allergen production between dog breeds. Contradictory results were observed for hair length ${ }^{4,5}$ and protein patterns. ${ }^{4,6}$ Breed-specific allergens have not been identified. ${ }^{4,6,7}$ Only 2 studies $^{6,8}$ have explored differences between levels of Can $\mathrm{f} 1$, a major dog allergen (Canisfamiliaris), ${ }^{4,9}$ in hair from different dog breeds. Variability in Can $\mathrm{f} 1$ levels between dog breeds was observed, but a higher variability between dogs of the same breed was seen. Poodles and Yorkshire terriers had the highest levels and Labrador retrievers had significantly lower allergen levels than other breeds. ${ }^{6,8}$

The reasons why symptoms of patients allergic to dogs could vary with different dog breeds are unclear. Exposure to allergens will be determined not only by the concentration in hair but also by shedding and other exposure-determining factors. ${ }^{10}$ Several nonshedding breeds, such as the Poodle and the Labradoodle, have been claimed to be and are marketed as hypoallergenic. The latter is a relatively new dog breed, first crossbred in Australia out of a Poodle and a Labrador retriever, with the goal of helping the blind with dog allergies. Only 1 study before looked at hypoallergenic dogs. In a human birth cohort study, Nicholas et al ${ }^{11}$ assigned owned dogs to different categories on the basis of being cited on the Web as hypoallergenic or not. No differences between these categories in Can $\mathrm{f} 1$ levels in floor dust from this birth cohort were found. Allergen levels in the dogs and differences between individual breeds were not considered.

No experimental evidence for the hypoallergenicity of any breed has yet been reported, nor for the amount of shedding of allergens in the environment and the resulting domestic exposure. In this study, relationships between Can $\mathrm{f} 1$ levels in dog hair and coat and in the home environment among various shedding and nonshedding breeds were investigated. The influence of dog breed and environmental characteristics on allergen levels was also compared.

\section{METHODS}

\section{Study population}

Dogs were recruited via breeders, breeder associations, and the companion animal hospital at the Faculty of Veterinary Medicine of Utrecht University, 


\author{
Abbreviations used \\ EDC: Electrostatic dust fall collector \\ GM: Geometric mean \\ GSD: Geometric standard deviation \\ LOD: Limit of detection \\ SAD: Settled airborne dust \\ SFD: Settled floor dust
}

the Netherlands. Six groups of dogs were included: Labradoodles $(n=114)$, Labrador retrievers $(n=54)$, Poodles $(n=45)$, Spanish Waterdogs $(n=13)$, Airedale terriers $(\mathrm{n}=24)$, and a control heterogeneous group $(\mathrm{n}=106)$ with 47 different non-hypoallergenic dog breeds and several crossbreds. For some analyses, the 6 groups were reduced to 2: hypoallergenic dogs (Labradoodles, Poodles, Spanish Waterdogs, and Airedale terriers; $n=196$ ) and nonhypoallergenic dogs (Labrador retrievers and the control group; $\mathrm{n}=160$ ) on the basis of anecdotal reports. ${ }^{11}$

In addition, a survey on dog allergy and allergic symptoms was conducted among the dog owners $(\mathrm{n}=502)$.

\section{Sample collection}

Sample collection was done by dog owners by using standardized protocols. Dog hair was collected by clipping the dogs' coat between the shoulders. Coat samples were collected by vacuuming one side of the dog for 30 seconds with a $25-\mu \mathrm{m}$ mesh nylon sample sock (Allied Filter Fabrics, Sydney, Australia) in the nozzle of the vacuum cleaner. Settled floor dust (SFD) samples of the living room floors were collected in a similar way, according to Schram-Bijkerk et al. ${ }^{12}$ Only homes with 1 dog were included. For 2 minutes, $1 \mathrm{~m}^{2}$ of a carpet or rug was vacuumed, or $2 \mathrm{~m}^{2}$ if there was a smooth floor or small rug $\left(<4 \mathrm{~m}^{2}\right)$. Passive airborne dust samples were collected in the living rooms (only in homes with $1 \mathrm{dog}$ ) by using the electrostatic dust fall collector (EDC) $)^{13,14}$ for 28 consecutive days, allowing the collection of settled airborne dust (SAD). Environmental samples were collected in the winter of 2010-2011. Participants were asked to complete questionnaires about their dog, house, and household. A translated version of all used questionnaires is available (see Fig E1 in this article's Online Repository at www.jacionline.org). All materials were returned by mail and stored at $4{ }^{\circ} \mathrm{C}$ (hair samples) or $-20^{\circ} \mathrm{C}$ (coat samples, SFD, and airborne dust) until extraction.

In addition to the sample collection, a short questionnaire (see Fig E1) on allergic symptoms was sent to all people 6 years and older living in the home of the dog (owners).

\section{Extraction}

Extraction was performed within 3 months after the collection of the samples. Between 110 and $130 \mathrm{mg}$ of the clipped hair was extracted with PBS, $0.25 \%$ azide, $\mathrm{pH}$ 7.20. Samples were incubated for 22 hours in the end-overend roller at $4^{\circ} \mathrm{C}$. Tubes were shaken, incubated in an ultrasonic bath, and spun at $3000 \mathrm{~g}$ at $4^{\circ} \mathrm{C}$ for 30 minutes. Vacuumed material from the dogs' coat was weighed and, depending on the net weight of the material, 2 to $8 \mathrm{~mL} \mathrm{PBS/}$ $0.25 \%$ azide $(<50 \mathrm{mg}, 2 \mathrm{~mL} ; 50-150 \mathrm{mg}, 4 \mathrm{~mL} ; 150-300 \mathrm{mg}, 6 \mathrm{~mL} ;>300$ $\mathrm{mg}, 8 \mathrm{~mL}$ ) was added. Hereafter, samples were treated in the same way as hair samples. Vacuumed floor dust was weighed prior to extraction. A total of $25 \mathrm{mg}$ of (representative) material was transferred into a tube and $2.5 \mathrm{~mL}$ PBS $/ 0.05 \%$ Tween 20 was added. Hereafter, allergen extraction was performed in accordance with Schram-Bijkerk et al. ${ }^{12}$ Allergen extraction of EDCs was performed as described previously. ${ }^{15}$ All supernatants were stored at $-20^{\circ} \mathrm{C}$ until analysis.

\section{Can $f 1$ assay}

Can $\mathrm{f} 1$ levels in supernatants were assayed by mAb-based ELISA (Indoor Biotechnologies, Charlottesville, Va) with polyclonal swine antirabbit immunoglobulins/horseradish peroxidase as conjugate (Dako, Glostrup, Denmark). The assay used purified natural Can $\mathrm{f} 1$ as standard, with protein content determined by amino acid analysis, as described previously. ${ }^{16,17}$ All results were based on duplicate measurements. Results were calculated back to $\mu \mathrm{g} / \mathrm{g}$ for hair, coat, and SFD or $\mu \mathrm{g} / \mathrm{m}^{2}$ for SAD and SFD.

Samples with values below the limit of detection (LOD) were given a value of two-third of the detection limit before backcalculation $(0.67 \mathrm{ng} / \mathrm{mL})$. The values below the LOD of the hair samples ranged from 0.02 to $0.12 \mu \mathrm{g} / \mathrm{g}$ and from 0.05 to $0.79 \mu \mathrm{g} / \mathrm{g}$ for coat samples. All SAD below the LOD were allotted a value of $0.64 \mu \mathrm{g} / \mathrm{m}^{2}$. Fifteen hair, 5 coat, $11 \mathrm{SAD}$, and no SFD samples had levels below the LOD.

\section{Statistical analysis}

Measured Can $\mathrm{f} 1$ concentrations were log-transformed. Analyses were performed by using SPSS (version 16, Chicago, Ill) for Windows to assess differences in Can $\mathrm{f} 1$ levels between breeds and the effect of dog and/or house characteristics on these levels. Geometric means (GMs) and geometric standard deviations (GSDs) were used to summarize results. Pearson correlations were used to assess associations between different samples. Multivariate linear regression analysis was used to study the influence of confounders.

\section{RESULTS \\ Dog owners}

Owners reported that $62 \%$ of the hypoallergenic dogs were selected for their marketed hypoallergenicity. In total, 502 owners of $168 \mathrm{dogs}$ filled in the questionnaire on allergic symptoms (see Table E1 in this article's Online Repository at www.jacionline. org). Three percent of the owners of non-hypoallergenic dogs and $22 \%$ of the owners of hypoallergenic dogs reported dog allergy. More than $80 \%$ of the allergic owners of hypoallergenic dogs reported experiencing fewer symptoms when in contact with hypoallergenic dogs compared with other dogs.

\section{Can $f 1$ levels in hair and coat samples}

Some samples were lost because of sampling errors or because there was not sufficient material for extraction. From $151 \mathrm{dogs}$, only hair samples were obtained (Table I). Log-transformed measured concentrations were normally distributed. Significantly higher $(P<.001)$ Can $\mathrm{f} 1$ levels were observed in hair of hypoallergenic breeds (GM, $2.26 \mu \mathrm{g} / \mathrm{g}$; GSD, 0.73) compared with nonhypoallergenic breeds (GM, $0.77 \mu \mathrm{g} / \mathrm{g}$; GSD, 0.71). There were significant differences between the different breeds, along with high variability in Can $\mathrm{f} 1$ levels between individual dogs of the same breed (Fig 1; Table II). Lowest allergen concentrations were found in hair of Labrador retrievers. Poodles had the highest concentrations and Labradoodles the second highest. Results were similar after adjustment in a multiple regression analysis for sex, age, and castration. Castrated dogs had a lower allergen load in hair, but this seemed mainly attributable to Labradoodles. Age showed a borderline significant $(P=.073)$ positive effect on Can $\mathrm{f} 1$ concentrations. No significant effects of sex, frequency of washing, recent washing ( $\leq 7$ days), or recent swimming ( $\leq 7$ days) on allergen levels were found.

Shedding of hair and dander, expressed as total weight (mg) of vacuumed coat material, was highest for Airedale terriers while it was similar for all other groups (Table II). Like in hair, Can $\mathrm{f} 1$ concentrations in the vacuumed coat material were significantly higher $(P<.001)$ for hypoallergenic breeds than for nonhypoallergenic breeds (GM, $27.04 \mu \mathrm{g} / \mathrm{g}, \mathrm{GSD}, 0.57$ and GM, 
TABLE I. Eligible study population, sample sizes, and dropouts with reasons for dropout

\begin{tabular}{lcccc}
\hline & \multicolumn{4}{c}{$\mathbf{n}(\%)$} \\
\cline { 2 - 5 } & Hair & Coat & SFD & SAD \\
\hline $\begin{array}{l}\text { Initially willing to } \\
\quad \text { participate }\end{array}$ & 473 & 322 & 247 & 247 \\
$\quad \begin{array}{l}\text { Not interested in } \\
\quad \text { participation anymore }\end{array}$ & $32(6.8)$ & $32(9.9)$ & $20(8.1)$ & $20(8.1)$ \\
$\quad$ Lost contact with & $48(10.1)$ & $48(14.9)$ & $35(14.2)$ & $35(14.2)$ \\
$\quad \begin{array}{l}\text { Error during mail contact } \\
\text { Error during collection or }\end{array}$ & $7(1.5)$ & $7(2.2)$ & $5(2.0)$ & $5(2.0)$ \\
$\quad$ extraction & & $20(6.2)$ & $14(5.7)$ & $4(1.6)$ \\
$\quad \begin{array}{l}\text { Excluded because of more } \\
\quad \text { dogs in the house }\end{array}$ & - & - & $12(4.9)$ & $15(6.1)$ \\
$\quad$ Usable samples & 351 & 215 & 161 & 168 \\
$\quad$ Labradoodle & $113(32.2)$ & $58(27.0)$ & $51(31.7)$ & $54(32.1)$ \\
$\quad$ Labrador retriever & $54(15.4)$ & $43(20.0)$ & $24(14.9)$ & $25(14.9)$ \\
$\quad \begin{array}{l}\text { Poodle } \\
\quad \text { Spanish Waterdog }\end{array}$ & $43(12.2)$ & $36(16.7)$ & $22(13.7)$ & $23(13.7)$ \\
$\quad$ Airedale terrier & $13(3.7)$ & $13(6.0)$ & $11(6.8)$ & $13(7.7)$ \\
$\quad$ Control group & $23(6.6)$ & $21(9.8)$ & $22(13.7)$ & $22(13.1)$ \\
$\quad 105(29.9)$ & $44(20.5)$ & $31(19.3)$ & $31(18.5)$ \\
\hline
\end{tabular}

$12.98 \mu \mathrm{g} / \mathrm{g}$, GSD, 0.76, respectively). Also, variability in coat samples showed the same pattern as in hair samples (Fig 1; Table II). Results were confirmed in a multiple regression model adjusting for sex, age, and castration. A significant positive effect of age was observed $(P=.028)$, while castration, sex, or washing were not associated with coat levels. Recent swimming appeared to reduce coat allergen levels across all breeds $(\beta=-0.328$; $P=.005)$. Moreover, differences in Can $\mathrm{f} 1$ levels between Labrador retrievers and Labradoodles and the control group diminished after adjustment for recent swimming. Allergen levels in hair and coat samples were positively correlated (Pearson $r^{2}$, $0.53 ; P<.001)$, but coat/hair ratios differed across breeds, indicating breed-dependent shedding. Higher ratios were found for Airedale terriers $(P=.002$, adjusted for sex, age, and castration status) and Poodles (not statistically significant).

\section{Can $f 1$ levels in environmental samples}

To explore differences in allergen distribution between different dog breeds, allergen levels in SFD and in air (SAD) were explored in homes of different dog breeds. Dust samples from houses with more than 1 dog were excluded from analyses (Table I). Log-transformed measured concentrations showed normal distribution.

SFD levels expressed in $\mu \mathrm{g} / \mathrm{g}$ did not differ between houses with hypoallergenic dogs and non-hypoallergenic dogs; however, Can $\mathrm{f} 1$ concentrations in floor dust of Labradoodle owners were significantly lower than in houses of other breeds $(P<.05$; Fig 2 ; Table II). Overall, the same pattern was seen when the concentration was expressed in $\mu \mathrm{g} / \mathrm{m}^{2}$ (Table II). The difference between Labradoodles and other dogs could not be explained by castration, sex, age, time spent indoors by the dog, washing or swimming of the dog, cleaning frequency of the house, or type of floor cover. As expected, Can $\mathrm{f} 1$ concentrations were considerably higher in rugs and carpets than in smooth floors $(P<.001)$. Can $f 1$ levels in floor dust samples expressed in $\mu \mathrm{g} / \mathrm{g}$ were only weakly correlated with concentrations in hair and coat samples (Pearson $r^{2}$, $0.20, P=.012$ and Pearson $r^{2}, 0.32, P<.001$, respectively).
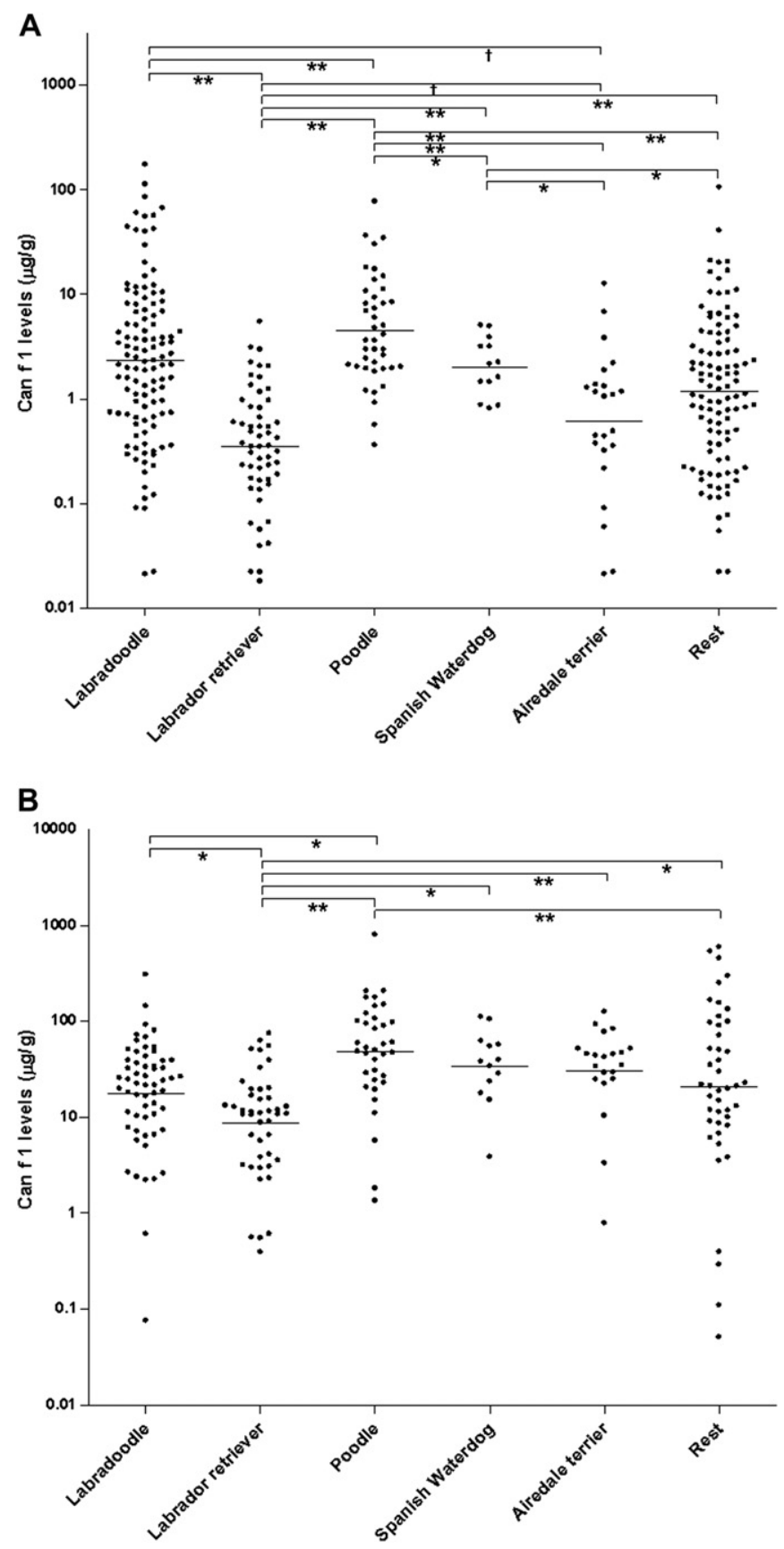

FIG 1. Can $f 1$ levels in hair (A) and coat (B) samples. Differences between breeds were tested for statistical significance in a multiple regression analysis adjusted for castration, sex, and age. $\dagger P<.1 ;{ }^{*} P<.05$; ${ }^{*} P<.001$.

When SFD levels of Can $\mathrm{f} 1$ were expressed in $\mu \mathrm{g} / \mathrm{m}^{2}$, there was a significant correlation only with coat levels (Pearson $r^{2}$, $0.20 ; P=.017)$.

Can $\mathrm{f} 1$ concentrations in airborne samples (SAD) from houses with a completely smooth floor were higher than in samples from houses with a rug. SAD levels did not differ significantly between hypoallergenic and non-hypoallergenic dogs, nor between the 6 breeds (Fig 2; Table II). Although allergen levels were influenced by the exposure period of EDCs (in days; mean, 28.5 days; range, 14-45 days; $P=.039$ ), type of floor coverage (smooth or a carpet or rug; $P=.034$ ), castration status (lower for houses with 
TABLE II. Population characteristics of the dogs enrolled and Can $\mathrm{f} 1$ levels for different sample types according to breed

\begin{tabular}{|c|c|c|c|c|c|c|}
\hline & Labradoodle & Labrador retriever & Poodle & Spanish Waterdog & Airedale terrier & Control group \\
\hline \multicolumn{7}{|l|}{ Population characteristics } \\
\hline $\mathrm{N}$ & 114 & 54 & 45 & 13 & 24 & 106 \\
\hline Age (mo), mean (range) & $25.7(1-110)$ & $57.0(8-155)$ & $49.2(2-196)$ & $53.5(9-105)$ & $44.5(6-150)$ & $74.7(1-194)$ \\
\hline Males (\%) & 30 & 48 & 58 & 54 & 67 & 57 \\
\hline Castrated (\%) & 56 & 50 & 56 & 46 & 42 & 52 \\
\hline \multicolumn{7}{|l|}{ Measurements } \\
\hline Can $\mathrm{f} 1$ in hair $(\mu \mathrm{g} / \mathrm{g}), \mathrm{GM}(\mathrm{GSD})$ & $2.32(0.77)$ & $0.35(0.56)$ & $4.43(0.51)$ & $2.03(0.28)$ & $0.61(0.71)$ & $1.16(0.72)$ \\
\hline $\begin{array}{l}\text { Total weight vacuumed coat material } \\
(\mu \mathrm{g} / \mathrm{g}), \mathrm{GM}(\mathrm{GSD})\end{array}$ & $19.36(0.42)$ & $26.49(0.50)$ & $20.56(0.56)$ & $37.58(0.32)$ & $59.98(0.57)$ & $25.41(0.55)$ \\
\hline Can $\mathrm{f} 1$ in coat $(\mu \mathrm{g} / \mathrm{g}), \mathrm{GM}(\mathrm{GSD})$ & $17.44(0.58)$ & $8.61(0.55)$ & $47.77(0.55)$ & $33.88(0.39)$ & $30.47(0.50)$ & $20.64(0.90)$ \\
\hline Can $\mathrm{f} 1$ in SFD $(\mu \mathrm{g} / \mathrm{g})$, GM (GSD) & $16.93(0.64)$ & $36.66(0.55)$ & $36.92(0.58)$ & $52.50(0.53)$ & $36.49(0.43)$ & $37.11(0.49)$ \\
\hline Can $\mathrm{f} 1$ in SFD $\left(\mu \mathrm{g} / \mathrm{m}^{2}\right), \mathrm{GM}(\mathrm{GSD})$ & $5,767.53(1.07)$ & $13,381.35(0.99)$ & $8,891.60(0.79)$ & $29,901.30(0.85)$ & $17,177.50(0.82)$ & $8,274.09(0.97)$ \\
\hline Can $\mathrm{f} 1$ in $\mathrm{SAD}\left(\mu \mathrm{g} / \mathrm{m}^{2}\right), \mathrm{GM}(\mathrm{GSD})$ & $5.22(0.40)$ & $5.04(0.50)$ & $6.32(0.47)$ & $9.05(0.59)$ & $8.44(0.35)$ & $7.18(0.56)$ \\
\hline
\end{tabular}

castrated dogs; $P=.009)$, and time the dog spent in the living room (in hours per day; $P<.001$, positive effect), these factors had no effect on the results between breeds in a regression analysis. No significant effects were observed for the time the dog had lived in the house (in months), washing or swimming of the dog, or cleaning frequency of the house. Can $\mathrm{f} 1$ concentrations in EDCs did not correlate with the other samples.

\section{DISCUSSION}

Our study showed that hypoallergenic dog breeds had higher Can $\mathrm{f} 1$ levels in their hair and coat than did non-hypoallergenic breeds, but these differences were not found in environmental exposure measurements. No earlier studies comparing hair, coat, and environmental levels of Can $\mathrm{f} 1$ in hypoallergenic and nonhypoallergenic dog breeds have been reported.

Hypoallergenicity is not an official breed characteristic of dogs, although many kennel clubs publish lists with breeds that are claimed to be hypoallergenic. Although the Labradoodle is not an officially recognized breed, it is the most well-known dog breed specifically crossbred with the aim of creating an "allergy-friendly" dog. On inquiry, it appeared that owners of hypoallergenic dogs enrolled in this study often purchased their hypoallergenic dog to ameliorate symptoms of dog allergies in their family. Allergic owners also reported fewer allergic symptoms to hypoallergenic dogs than to non-hypoallergenic dogs. Results of the owners should be interpreted with care as the study was not designed to analyze reduced allergic symptoms in owners. In addition, participants were not blinded and answers may be subject to selective recall. Results are only shown to illustrate the alleged hypoallergenicity of certain breeds.

Dog hair and dander are major sources of Can $\mathrm{f} 1,{ }^{6,9}$ and the allergen was detectable in almost all hair and coat samples. Saliva is an important source of Can $\mathrm{f} 1$ as well ${ }^{18,19}$ although this was not studied. In literature, other dog allergens such as dog serum albu$\min \left(\operatorname{Can} \mathrm{f} 3^{20}\right)$, Can f $2,{ }^{19}$ and Prostatic kallikrein $\left(\right.$ Can $\mathrm{f} 5^{21}$ ) are described as well, but until now no techniques to measure these allergens in large studies are available. It cannot be excluded that differences exist in exposure in these allergens between the different breeds.

Although Can f 1 levels in hair and coat samples were significantly related to breed, enormous variability (up to 4 orders of magnitude) was observed between individual dogs of the same breed. This may reflect differences between individuals but might also be influenced by less obvious factors such as grooming habits. Significantly higher Can $\mathrm{f} 1$ levels were found in hair and coat samples of hypoallergenic breeds than of non-hypoallergenic breeds. The findings on Labrador Retrievers and Poodles are in agreement with an earlier study that compared Can $\mathrm{f} 1$ levels in different breeds but did not focus on differences between hypoallergenic and non-hypoallergenic breeds. ${ }^{8}$ Coat and hair allergen levels were positively correlated, but differences between the groups seen in hair samples diminished in the coat samples. Coat allergen/hair allergen ratios differed per breed and were particularly high for Airedale terriers. Different coat/hair ratios may reflect differences in the availability of allergens in the coat.

Despite differences in hair and coat levels between hypoallergenic and non-hypoallergenic dogs, no differences were observed in environmental levels, which is in agreement with Nicholas et al. ${ }^{11}$ In the present study, Can $\mathrm{f} 1$ levels in SFD from houses of Labradoodles were significantly lower than from houses of other breeds. However, in the airborne dust, this difference was not found. Although owners' self-reported cleaning frequencies did not differ, it is possible that owners of hypoallergenic dogs, who were more often allergic, have other cleaning habits or clean more thorough. They may have high-efficiency particle arrest filters in their vacuum cleaners more often or have other grooming habits that influenced the SFD levels but not the SAD levels. Other factors that could have influenced environmental results are the socioeconomic status of the owner, previous dog ownership as well as other house characteristics not reported. Although influences are expected to be small, they cannot be excluded.

The EDC is a low-cost and easily applicable method to measure airborne settled dust compared with measuring airborne dust actively. It is placed at $1.5 \mathrm{~m}$ or higher so that only airborne dust can be settled on it. The technique correlates moderately to well with active airborne sampling. ${ }^{14,15}$ It is difficult to compare the SAD levels with other studies due to different techniques used. Only 1 study on dog allergens was performed with the EDC before, ${ }^{15}$ which measured Can $\mathrm{f} 1$ in an animal clinic. Although that was a different setting, found levels of airborne exposure were in the same range.

No correlation between SFD and SAD levels was found. Can $\mathrm{f}$ 1 is mainly found in large dust particles $(>9 \mu \mathrm{m})$, which deposit fairly quick. Approximately $20 \%$ of the airborne allergen load is carried on particles of aerodynamic size $(<5 \mu \mathrm{m})$ that are capable 

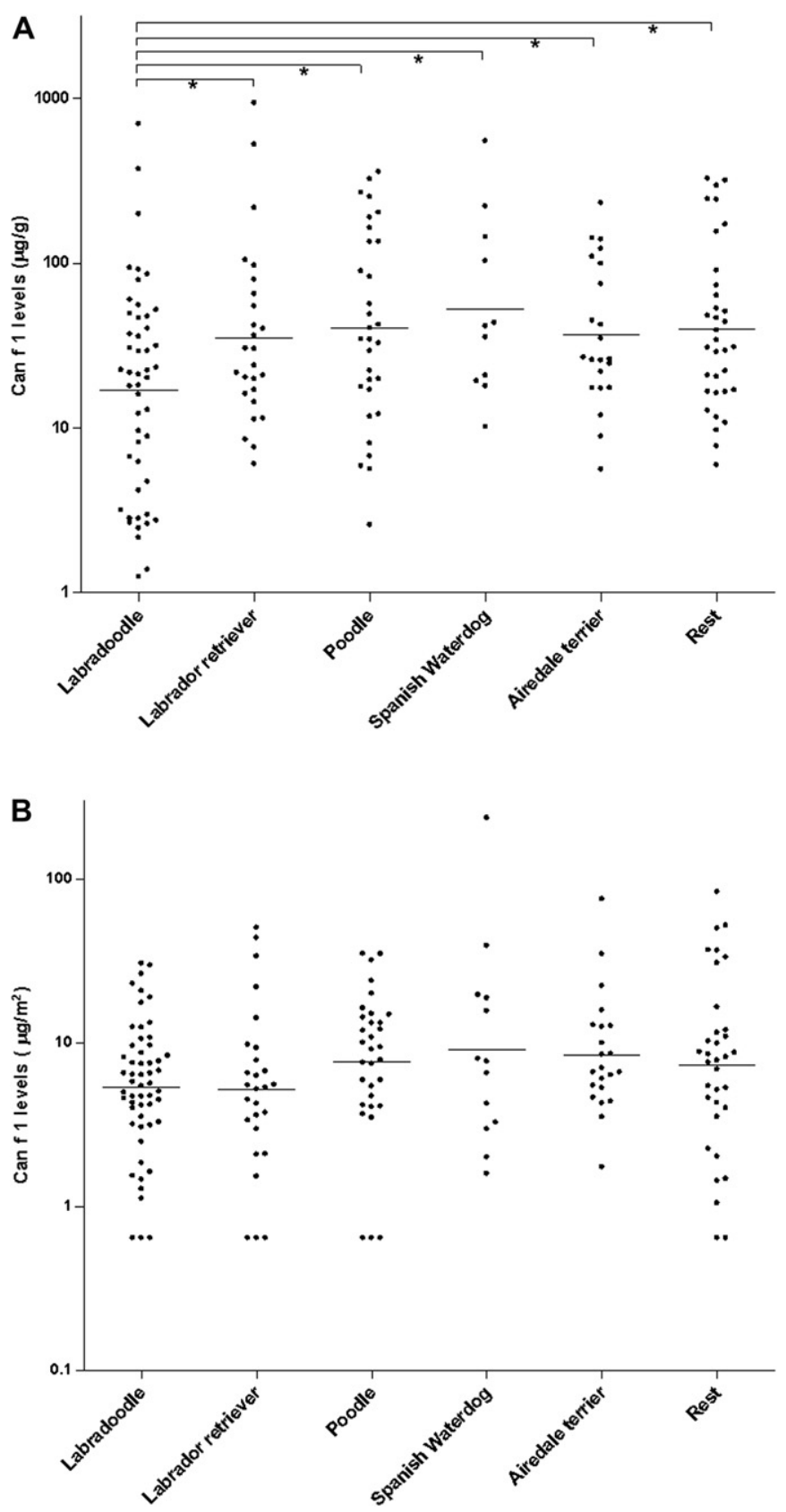

FIG 2. Can $f 1$ levels in SFD (A) and SAD (B) samples. Differences between breeds were tested for statistical significance in a multiple regression analysis adjusted for type of floor (Fig 2, $A$ ) and sampling duration (days), type of floor, castration status of the dog, and time spent indoors by the dog (hours per day) (Fig 2, B). ${ }^{*} P<.05$.

of remaining airborne for longer periods. ${ }^{22}$ It is expected that airborne allergen levels reflect personal allergen exposure better than do SFD levels. ${ }^{15}$ However, it is an often-used technique and in addition to the coat samples it can provide information on shedding. It is difficult to compare these results with the results of previous studies as techniques for dust collection ${ }^{23}$ and measuring allergens ${ }^{16,17}$ can vary. However, SFD levels found in this study are 10 times higher than those found in child bedrooms ${ }^{10,11}$ but are in the same range as levels found in living rooms ${ }^{3}$ and in an animal clinic, ${ }^{15}$ and are well above the suggested thresholds for symptoms. ${ }^{2,23}$
Castration was clearly associated with lower air levels, while floor dust levels were not influenced. Castration also was associated with reduced Can f levels in hair. Thus, interestingly, shedding, as represented by coat levels, was not different between castrated and noncastrated dogs. The differences in air levels could be associated with the observed lower hair levels or with differences in the activity patterns of castrated dogs, but this requires further confirmation. Recent swimming appeared to be a strong determinant for Can $\mathrm{f}$ levels in coat samples. This indicates that swimming reduces the amount of dander. Ramadour et $\mathrm{al}^{8}$ suggested that low allergen concentrations in hair of Labrador retrievers may be caused by their affinity for swimming. Indeed, the prevalence of recent swimming in our study was higher for Labrador retrievers. Contradictory to Hodson et al, ${ }^{24}$ in our study, recent washed dogs $(n=19)$ did not have lower allergen concentrations in their hair, coat, or environment than other dogs.

In conclusion, this work showed for the first time that Can $\mathrm{f}$ 1 levels in hair and coat samples are significantly related to breed, although high variability within breeds is observed. Significantly higher Can $\mathrm{f} 1$ levels in hair and coat samples were found in dog breeds that were considered hypoallergenic than in other breeds while airborne Can $\mathrm{f} 1$ levels in homes were similar across different breeds. No evidence was found for a reduced production of allergen by hypoallergenic dogs. In addition, no evidence for reduced shedding of allergens to the coat as well as to the dogs' living environment was found. These results show that even reportedly hypoallergenic dog breeds are responsible for levels of environmental allergen exposure that have been associated with allergic reactions and asthma. In conclusion, the term "hypoallergenic" is a misnomer that is not evidence based and should not be applied to dog breeds on the basis of current scientific evidence.

We thank the dog owners, the dog breeders, Ron van Wandelen, and Max Verver for their participation. Jack Spithoven and Siegfried de Wind are acknowledged for their laboratory assistance.

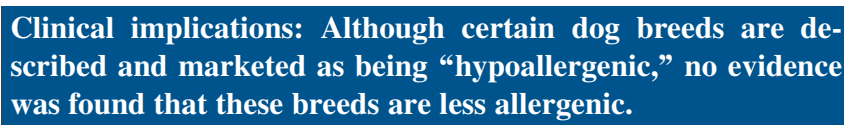

\section{REFERENCES}

1. Custovic A, Simpson BM, Simpson A, Hallam CL, Marolia H, Walsh D, et al. Current mite, cat, and dog allergen exposure, pet ownership, and sensitization to inhalant allergens in adults. J Allergy Clin Immunol 2003;111:402-7.

2. Ingram JM, Sporik R, Rose G, Honsinger R, Chapman MD, Platts-Mills TA. Quantitative assessment of exposure to $\operatorname{dog}$ (Can f 1) and cat (Fel d 1) allergens: relation to sensitization and asthma among children living in Los Alamos, New Mexico. J Allergy Clin Immunol 1995;96:449-56.

3. Langley SJ, Goldthorpe S, Craven M, Morris J, Woodcock A, Custovic A. Exposure and sensitization to indoor allergens: association with lung function, bronchial reactivity, and exhaled nitric oxide measures in asthma. J Allergy Clin Immunol 2003; 112:362-8

4. Blands J, Lowenstein H, Weeke B. Characterization of extract of dog hair and dandruff from six different dog breeds by quantitative immunoelectrophoresis: identification of allergens by crossed radioimmunoelectrophoresis (CRIE). Acta Allergol 1977;32:147-69.

5. Hooker SB. Qualitative differences among canine danders. Ann Allergy 1944;2: 281.

6. Heutelbeck AR, Schulz T, Bergmann KC, Hallier E. Environmental exposure to allergens of different dog breeds and relevance in allergological diagnostics. J Toxicol Environ Health A 2008;71:751-8.

7. Lindgren S, Belin L, Dreborg S, Einarsson R, Pahlman I. Breed-specific dog-dandruff allergens. J Allergy Clin Immunol 1988;82:196-204. 
8. Ramadour M, Guetat M, Guetat J, El Biaze M, Magnan A, Vervloet D. Dog factor differences in Can f 1 allergen production. Allergy 2005;60:1060-4.

9. Schou C, Svendsen UG, Lowenstein H. Purification and characterization of the major dog allergen, Can f I. Clin Exp Allergy 1991;21:321-8.

10. Nicholas C, Wegienka G, Havstad S, Zoratti E, Ownby D, Johnson CC. Dog characteristics and allergen levels in the home. Ann Allergy Asthma Immunol 2010; 105:228-33

11. Nicholas CE, Wegienka GR, Havstad SL, Zoratti EM, Ownby DR, Johnson CC Dog allergen levels in homes with hypoallergenic compared with nonhypoallergenic dogs. Am J Rhinol Allergy 2011;25:252-6.

12. Schram-Bijkerk D, Doekes G, Boeve M, Douwes J, Riedler J, Ublagger E, et al. Exposure to microbial components and allergens in population studies: a comparison of two house dust collection methods applied by participants and fieldworkers. Indoor Air 2006;16:414-25.

13. Noss I, Doekes G, Sander I, Heederik DJ, Thorne PS, Wouters IM. Passive airborne dust sampling with the electrostatic dustfall collector: optimization of storage and extraction procedures for endotoxin and glucan measurement. Ann Occup Hyg 2010;54:651-8.

14. Noss I, Wouters IM, Visser M, Heederik DJ, Thorne PS, Brunekreef B, et al. Evaluation of a low-cost electrostatic dust fall collector for indoor air endotoxin exposure assessment. Appl Environ Microbiol 2008;74:5621-7.

15. Samadi S, Heederik DJ, Krop EJ, Jamshidifard AR, Willemse T, Wouters IM. Allergen and endotoxin exposure in a companion animal hospital. Occup Environ Med 2010;67:486-92.
16. Filep S, Tsay A, Vailes L, Gadermaier G, Ferreira F, Matsui E, et al. A multiallergen standard for the calibration of immunoassays: CREATE principles applied to eight purified allergens. Allergy 2012;67:235-41.

17. Filep S, Tsay A, Vailes LD, Gadermaier G, Ferreira F, Matsui E, et al. Specific allergen concentration of WHO and FDA reference preparations measured using a multiple allergen standard. J Allergy Clin Immunol 2012;129:1408-10.

18. Ford AW, Alterman L, Kemeny DM. The allergens of dog, I: identification using crossed radio-immunoelectrophoresis. Clin Exp Allergy 1989;19:183-90.

19. Kamata Y, Miyanomae A, Nakayama E, Miyanomae T, Tajima T, Hoshi H. Characterization of $\operatorname{dog}$ allergens Can $\mathrm{f} 1$ and Can $\mathrm{f} 2$. 1: preparation of their recombinant proteins and antibodies. Int Arch Allergy Immunol 2007;142:291-300.

20. Boutin Y, Hebert H, Vrancken ER, Mourad W. Allergenicity and cross-reactivity of cat and dog allergenic extracts. Clin Allergy 1988;18:287-93.

21. Mattsson L, Lundgren T, Everberg H, Larsson H, Lidholm J. Prostatic kallikrein: a new major dog allergen. J Allergy Clin Immunol 2009;123:362-8.

22. Custovic A, Green R, Fletcher A, Smith A, Pickering CA, Chapman MD, et al Aerodynamic properties of the major $\operatorname{dog}$ allergen Can $\mathrm{f} 1$ : distribution in homes, concentration, and particle size of allergen in the air. Am J Respir Crit Care Med 1997; 155:94-8

23. Wilson J, Dixon SL, Breysse P, Jacobs D, Adamkiewicz G, Chew GL, et al. Housing and allergens: a pooled analysis of nine US studies. Environ Res 2010;110:189-98.

24. Hodson T, Custovic A, Simpson A, Chapman M, Woodcock A, Green R. Washing the dog reduces dog allergen levels, but the dog needs to be washed twice a week. J Allergy Clin Immunol 1999;103:581-5. 


\section{PART 1 \\ (please complete on day 1)}

1. Name:

2. Place of residence:

3. Date:

4. For how long has your dog been living with you in this house?

5. How much time, on average, does your dog spend in the living room per day (24 hours)?

6. Do you own other animals which may enter the living room?Yes/no

a. If 'yes': how many and of which species?

7. Did you purchased this specific dog breed in consideration with dog allergies within your family?

o No

o Yes; please explain:

8. What is the year of construction of your house?

9. In what type of house do you live?
o Terrace house
o Corner house
- Apartment
- Semi-detached house
o Detached house
o Something else, namely:

10. What is the composition of your household (fill in the number of people living in your house)?
$<1$ year old
_ 1-12 years old
_ 13-18 years old
Adults

11. What type of flooring do you have in your living room?

o Carpet:

- Long pile/short pile

o Smooth floor with a rug:

- Long pile/short pile

- Rug size: $+/-\ldots . . \mathrm{cm} \times \ldots . . \mathrm{cm}$

- Smooth floor without a rug

o Something else, namely:

12. On what date did you start sampling with the EDC in your living room?

13. On what height did you set the EDC? $\mathrm{cm}$ 


\section{Thank you for completing this questionnaire!}

Fill in any comments:

FIG E1. This figure contains a translation (from Dutch) of the questionnaires used in the study. The questionnaire consists of 3 parts. The first part was filled in on the first day of the exposure assessment. The second part was filled in at the end of the exposure assessment period. These 2 parts contain questions on household characteristics, dog characteristics, and dog behavior. Part 3 is a health questionnaire. We asked all people living in the household (6 years or older) to fill in this questionnaire. The questionnaire was returned by mail together with the collected materials. 


\section{PART 2}

(please complete on day 28)

1. Date:

2. Breed of the dog:

3. Sex of the dog:

$\mathrm{m} / \mathrm{f}$

4. Is he/she neutered?

Yes/no

5. Date of birth of the dog:

6. Date of last heat (if applicable):

7. Date of last nest (if applicable):

8. Do you brush your dog?

$$
\text { Yes/no }
$$

a. If 'yes': with what frequency?

b. Date of last time:

9. Do you wash your dog? Yes/no

a. If 'yes': with what frequency?

b. Date of last time:

10. Does your dog swim?

Yes/no

a. If 'yes': date of last time:

11. Has your dog had any problems with his/her coat or skin in the last 6 months? Yes/no

a. If 'yes', please explain:

If you have any additional comments on the health status of your dog please write below:

12. How frequently do you clean your living room?

\begin{tabular}{|c|c|c|c|c|c|}
\hline & Daily & $\begin{array}{c}2 \text { to } 4 \\
\text { times a } \\
\text { week }\end{array}$ & $\begin{array}{l}\text { Once a } \\
\text { week }\end{array}$ & $\begin{array}{c}\text { Less than } \\
\text { once a } \\
\text { week }\end{array}$ & Never \\
\hline With the broom & $\square$ & $\square$ & $\square$ & $\square$ & $\square$ \\
\hline With the vacuum cleaner & $\square$ & $\square$ & $\square$ & $\square$ & $\square$ \\
\hline With the mop & $\square$ & $\square$ & $\square$ & $\square$ & $\square$ \\
\hline $\begin{array}{l}\text { Something else, namely: } \\
\ldots \ldots \ldots \ldots \ldots \ldots \ldots \ldots \ldots \ldots \ldots \ldots \ldots \ldots \ldots \ldots \ldots \ldots \ldots \ldots \ldots \ldots\end{array}$ & $\square$ & $\square$ & $\square$ & $\square$ & $\square$ \\
\hline
\end{tabular}

13. On what date have you cleaned the floor of your living room for the last time?

14. In the past 28 days, did any other dog than your own dog visit your living room? Yes/no 
If 'yes':

a. How many?

b. For how long?

15. Could you please, like in the example below, sketch the floor plan of your living room and indicate:

a. Where you placed the EDC

b. Where you took the floor dust sample

Example:

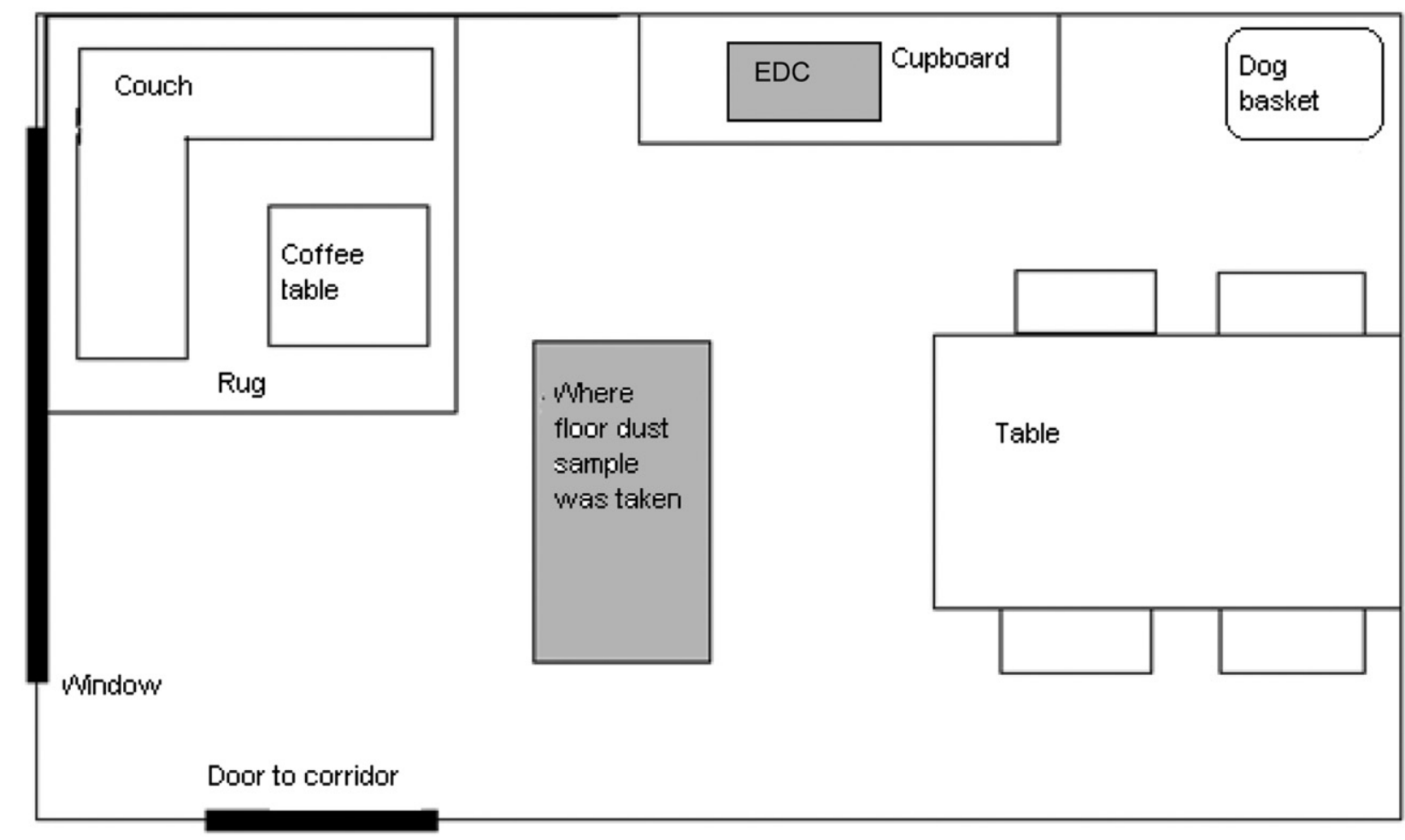

Your living room:

16. On what date did you close the EDC?

17. Please note below any details that have occurred with respect to the dust sample taking with the EDC (for example: EDC touched by a pet, EDC moved or fell down):

18. On what date did you take the other samples:

19. Please note below any details that have occurred with respect to the sample taking (for example: different dimensions of the vacuumed surface, dust fell out of the sample sock, different hair sample location):

Thank you for completing this questionnaire! If you have any comments please write below: 


\section{PART 3}

\section{(please complete for every family member above the age of 5)}

1. Sex:

2. Age:

3. Have you had wheezing or whistling in your chest in the last 12 months when you did not have a cold? Yes/no

4. Have you woken up with a feeling of tightness in your chest at any time in the last 12 months?

$$
\text { Yes/no }
$$

5. Have you had an attack of shortness of breath that came on during the day when you were at rest at any time in the last 12 months? Yes/no

6. Have you had an attack of shortness of breath that came on following strenuous activity at any time in the last 12 months? Yes/no

7. Have you been woken by an attack of coughing at any time in the last 12 months?

$$
\text { Yes/no }
$$

8. Have you ever had asthma? Yes/no

If 'yes':

a. Was this confirmed by a doctor? Yes/no

b. Have you had an attack of asthma in the last 12 months? Yes/no

9. Have you ever had a problem with sneezing, or a runny or blocked nose when you did not have a cold or flu? Yes/no

If 'yes':

a. Has this nose problem been accompanied by itchy or watery eyes? Yes/no

b. Have you had a problem with sneezing, or a runny or blocked nose when you did not have a cold or flu in the last 12 months? Yes/no

10. Do you have any nasal allergies? Yes/no

If 'yes':

a. What type of allergies do you have?

o Dust mites

o Hay fever

o Dogs

- Cats

o Something else, namely:

b. Was this confirmed by a doctor? 
11. Do you experience allergy related symptoms during or following contact with dogs? Yes/no

a. If 'yes':

a1. What is the severity of these symptoms to your own dog?
o Moderate
- Average
o Severe

a2. What is the severity of these symptoms to other dogs?
o Moderate
o Average
o Severe

a3. Do you experience less or more severe allergy related symptoms to a specific dog breed? Yes/no

If 'yes', please explain:

Thank you for completing this questionnaire!

If you have any comments please write below: 
TABLE E1. Asthmatic and allergic symptoms of dog owners

All participating dog owners

Non-hypoallergenic dog owner

Hypoallergenic dog owner

Participating dog owners (male/female)

Mean age (y), range

Mean number of participating owners per dog

Self-reported asthma, n (\%)

Self-reported allergy (rhinitis), n (\%)

Self-reported dog allergy, n $(\%)$

Dog-allergic owners who reported fewer allergic symptoms in contact with hypoallergenic dog compared with other dogs, n (\%)

$502(241 / 261)$
$35(6-78)$
2.99
$94(19)$
$178(35)$
$83(17)$
$65(78)$

$146(68 / 78)$

$39(7-78)$

2.65

$12(8)$

39 (27)

$5(3)$

$0(0)$

$356(173 / 183)$

33 (6-71)

3.15

82 (23)

139 (39)

78 (22)

65 (83) 